\title{
Efficacy and Safety of Novel Formulation of Clobetasol Propionate $0.025 \%$ Cream in Indian Moderate-to- Severe Psoriasis Patients: Phase-2a, Randomized 3-Arm Study
}

\author{
Srinivas Sidgiddi · Syed Mujtaba Hussain Naqvi • Manjunath Shenoy • \\ Devang Narayan Balraj · Jayesh Kothari · Sandesh Gupta • \\ Rizwan Haq · Rajan Mittal · Suyog Mehta · Amey Mane
}

Received: February 22, 2021 / Published online: August 28, 2021

(C) The Author(s) 2021

\section{ABSTRACT}

Introduction: Clobetasol propionate $(0.05 \%$ standard dose formulation), a topical corticosteroid, leads to systemic side-effects like hypothalamic-pituitary-adrenal (HPA) axis

Supplementary Information The online version contains supplementary material available at https:// doi.org/10.1007/s13555-021-00591-z.

S. Sidgiddi

Clinical Development, Dr. Reddy's Laboratories

Inc., Princeton, USA

S. M. H. Naqvi ( $₫)$ · R. Mittal · S. Mehta · A. Mane Medical Affairs, Dr. Reddy's Laboratories Pvt Ltd, 27, Ameerpet Rd, Leelanagar, Hyderabad, Telangana 500016, India

e-mail: mujtabanaqvi@drreddys.com

\section{Shenoy}

Department of Dermatology, Yenepoya Medical College, Yenepoya University, Mangalore, India

\section{N. Balraj}

Department of Dermatology, Rajbal Skin Clinic, Bangalore, India

J. Kothari

Department of Dermatology, Kothari Skin Clinic, Indore, India

\section{S. Gupta}

Department of Dermatology, Skin and Laser Center, New Delhi, India

R. Haq

Department of Dermatology, Radiance Skin Clinic, Nagpur, India suppression at doses as low as $2 \mathrm{~g} /$ day. The aim of this study was to evaluate HPA axis suppression, efficacy, and safety of clobetasol propionate $(0.025 \%$, formulation 5 and 13$)$ versus currently marketed $0.05 \%$ cream in Indian patients with moderate-to-severe psoriasis.

Methods: In this phase 2a investigator-blinded study, patients aged $\geq 18$ years with moderateto-severe psoriasis were randomized 1:1:1 to receive clobetasol propionate $0.025 \%$ formulation 5 , or 13 , or $0.05 \%$ cream; twice daily for 28 days. Safety endpoints included adrenocorticotropic hormone (ACTH) test results at day 28 (primary), and local tolerability at each visit (burning/stinging/pruritus, secondary). Efficacy endpoints included Psoriasis Global Assessment (PGA) score.

Results: Overall, 88 patients received clobetasol propionate $0.025 \%$ formulation 5 and 13 $(n=29$ for both) and $0.05 \%$ cream $(n=30)$. At day 28 , the proportion of patients with an abnormal ACTH stimulation test (cortisol levels $\leq 18 \mu \mathrm{g} / \mathrm{dl}$ ) was numerically lower in $0.025 \%$ formulations: 5 (20.7\%) and 13 (17.2\%) compared with $0.05 \%$ cream (30.0\%), $(p=0.320)$. Decrease in burning/stinging /pruritus scores were comparable in all treatment groups and PGA success rates were higher with $0.025 \%$ formulations: 5 (38.9\%) and 13 (36.8\%) compared with $0.05 \%$ cream $(30.8 \%)$.

Conclusion: Clobetasol propionate $0.025 \%$ could be an effective treatment for moderate-tosevere psoriasis compared with $0.05 \%$ cream, 
demonstrating comparable efficacy with a better systemic safety profile.

Trial Registration Number: REF/2018/01/ 016779.

Keywords: Clobetasol propionate $0.025 \%$; Clobetasol propionate $\quad 0.05 \%$; Hypothalamic-pituitary-adrenal axis suppression; Moderate-to-severe psoriasis; Psoriasis Global Assessment; Novel formulation

\section{Key Summary Points}

We aimed to evaluate and compare hypothalamic-pituitary-adrenal (HPA) axis suppression, safety and efficacy of two novel formulations of clobetasol propionate $0.025 \%$ cream (formulation 5 $\left[\right.$ Impoyz $\left.{ }^{\circledR}\right]$ and formulation 13) with the currently marketed clobetasol propionate $0.05 \%$ emollient cream (reference formulation) in Indian patients with moderate-to-severe psoriasis.

Two clobetasol formulations, formulation 5 and formulation 13, has similar efficacy and an improved systemic safety profile compared to the clobetasol propionate $0.05 \%$ (Temovate $\mathrm{E}^{\circledR}$ ) cream in terms of HPA axis suppression.

Our study findings support the use of novel clobetasol propionate $0.025 \%$ cream formulations (formulation 5 and formulation 13) as an effective and safer treatment option for patients with moderate-to-severe psoriasis.

\section{INTRODUCTION}

Psoriasis is a chronic autoimmune skin disorder affecting approximately $0.44-2.8 \%$ of the adult population in India [1-3]. The prevalence of psoriasis varies across countries from $0.09-11.43 \%$, leading to a serious global burden with a significant negative impact on patients' quality of life [4]. The symptoms of psoriasis may occur anywhere on the body and are often characterized by chronic, sharply demarcated, and erythematous scaly plaques that are often painful and severely pruritic [2, 5]. Although not life threatening, these persistent symptoms affect physical, psychological, and social functioning and remain a major challenge to treat in patients with disease complications $[6,7]$. The majority of the patients affected with mild [Psoriasis Area Severity Index (PASI) $\leq 10$ ] to moderate (PASI $>10$ but $\leq 20$ ) psoriasis are generally recommended for treatment with topical therapies such as corticosteroids and vitamin D3 analogs [2, 8, 9]. The systemic therapies including phototherapy, acitretin, methotrexate, cyclosporine, or biologic therapy are recommended for severe psoriasis. However, patients on systemic or phototherapies are adjunctively treated with topical therapies based on the psoriasis severity, body location, lesion thickness, degree of erythema, and amount of scaling $[6,9,10]$. Topical corticosteroids are the most commonly prescribed medication for patients with moderate-to-severe psoriasis and several different types of formulations are currently available [11]. Clobetasol propionate, an ultra-high potent (super potent) topical corticosteroid compared with other corticosteroids, has demonstrated effective and rapid healing of psoriasis [12-16].

The super-high potency of clobetasol propionate $0.05 \%$ emollient cream leads to greater systemic absorption, which might result in higher risk of systemic side-effects such as hypothalamic-pituitary-adrenal axis (HPA) suppression, even at the recommended dose as low as $2 \mathrm{~g} /$ day. This systemic side-effect has generally limited the clinical use of clobetasol propionate $0.05 \%$ cream in patients to small areas and only for a single period not exceeding 2 weeks [15]. Therefore, there is an unmet need to develop novel formulations containing $0.025 \%$ concentrations of clobetasol propionate, which would provide a favorable benefitrisk profile. In a recent phase 2 , randomized, open-label clinical study conducted in the US, treatment with clobetasol propionate $0.025 \%$ $\left(\right.$ Impoyz $^{\circledR}$ ) cream had a lower systemic absorption, resulting in lower incidence of HPA axis suppression, with a better safety profile compared with marketed clobetasol propionate 
$0.05 \% \quad$ Temovate ${ }^{\circledR}$ ) cream in patients with moderate-to-severe psoriasis [17].

In this study, we aimed to evaluate and compare HPA axis suppression, safety, and efficacy of two formulations of clobetasol propionate $0.025 \%$ cream [formulation 5 (Impoyz ${ }^{\circledR}$ ) and formulation 13] with the currently marketed clobetasol propionate $0.05 \%$ emollient cream (reference formulation) in Indian patients with moderate-to-severe psoriasis.

\section{METHODS}

\section{Patients}

The study included adults aged $\geq 18$ years with a clinical diagnosis of moderate-to-severe psoriasis involving at least $25 \%$ body surface area (BSA, excluding scalp, face, groin, axillae, and/ or other intertriginous areas involvement, if present). Other inclusion criteria were a Psoriasis Global Assessment (PGA) score of $\geq 3$ at screening and baseline, and no evidence of abnormal HPA axis function [confirmed by adrenocorticotropic hormone (ACTH) test] at screening.

Patients who were hypersensitive to clobetasol propionate, or who had a history of psoriasis that was unresponsive to topical corticosteroid therapy, or who had experienced adverse drug reaction to cosyntropin injection (adrenocorticitropic hormone) were excluded from the study. Patients who had a known history of acute adrenal crisis, Addison's disease, atopic/contact dermatitis, liver disease, renal impairment, heart disease, diabetes, severe respiratory disease, rheumatoid arthritis, malignancies, and immunocompromised conditions were also excluded from the study. Other exclusion criteria were pregnant/lactating women; patients who were treated with radiation therapy or anti-neoplastic agents, or immunosuppressants (within 4 weeks prior to study treatment), antipsoriatics (within 8 weeks of study treatment), biological therapies (within 12 weeks prior to study treatment), and topical corticosteroids (within 2 weeks prior to screening) to ensure complete washout of previously administered drugs to prevent drug interactions.

\section{Study Design}

This was a phase $2 \mathrm{a}$, randomized, multicenter, active-controlled, investigator-blinded, parallel group, 3-arm study of two formulations of clobetasol propionate $0.025 \%$ cream (formulations 5 and 13) and clobetasol propionate $0.05 \%$ cream, in patients with moderate-to-severe psoriasis. This study is registered as REF/2018/ 01/016779. The study was conducted to compare HPA axis suppression, safety, and efficacy of twice daily use of clobetasol propionate $0.025 \%$ formulations (formulations 5 and 13; manufactured by Dr. Reddy's Laboratories) with the reference formulation (clobetasol propionate $0.05 \%$ cream; manufactured by PharmaDerm). The study comprised a 7-day screening period, a 28 -day treatment schedule and a 14-day follow-up period.

The study protocol and informed consent form were reviewed and approved by an Independent Ethics Committee and Institutional Review Board at all six study sites (please see supplementary material for full details). The study was conducted in accordance with the ethical principles of the Declaration of Helsinki and per the International Council for Harmonization and Good Clinical Practice guidelines. Informed consent forms were reviewed and approved by all the appropriate Ethics Committees prior to enrolment of the patients into the study.

\section{Study Assessments}

The primary safety endpoint included proportion of patients with abnormal cortisol levels in response to the ACTH test $(\leq 18 \mu \mathrm{g} / 100 \mathrm{ml})$ at day 28. Secondary safety endpoints included time to abnormal cortisol value and change from baseline in signs of atrophogenicity at days $7,14,21$, and 28 . The local cutaneous atrophogenic activity of the drug was determined by the investigator using visual assessment at planned visits. The effect of the study medication was graded on a scale of 0 (none) to 
3 (pronounced) for atrophy and 0 (none) to 4 (pronounced) for telangiectasia. Adverse events (AEs), physical examination, vital signs, clinical laboratory investigations, and local tolerability (rating of burning/stinging and pruritus by the patient) were also recorded. The degree of burning/stinging and pruritus were scored on a scale of 0 (none) to 3 (severe).

Secondary efficacy endpoints included proportion of patients with clear or almost clear skin as assessed by PGA, mean PGA score, and distribution of PGA scores at each time point. Clinical determination of disease severity using the PGA scale was performed by the investigator at planned visits. The PGA was scored with five severity grades on a scale of 0 (clear) to 4 (moderate-to-severe).

\section{Treatment, Randomization and Blinding}

Formulation 13 consisted of $0.025 \%$ clobetasol propionate with inactive ingredients like stearyl alcohol, cetyl alcohol, glyceryl stearate and PEG-100 stearate, lanolin, mineral oil, sorbitol, butylated hydroxytoluene, diethylene glycol monoethyl ether, methyl paraben, propyl paraben, and purified water. Formulation 5 consisted of $0.025 \%$ clobetasol propionate with inactive ingredients like cetostearyl alcohol, glyceryl stearate and PEG-100 stearate, white wax, butylated hydroxytoluene, isopropyl myristate, cyclomethicone, diethylene glycol monoethyl ether, methyl paraben, propyl paraben, and purified water.

Patients were randomized 1:1:1 (using PROC PLAN in SAS, version 9.2) to clobetasol propionate $0.025 \%$ formulation 5 , formulation 13 or clobetasol propionate $0.05 \%$ cream. Patients were instructed to topically apply the study medication twice daily for 28 days to the target lesion areas (at least 25\% BSA), which were identified by the study investigator. The study products were applied in the form of fingertip unit $(\sim 0.5 \mathrm{~g})$. Patients were asked to apply seven fingertip units in order to cover $25 \%$ BSA (3.5 g).

The treatments were blinded by obscuring the commercial labeling of comparator

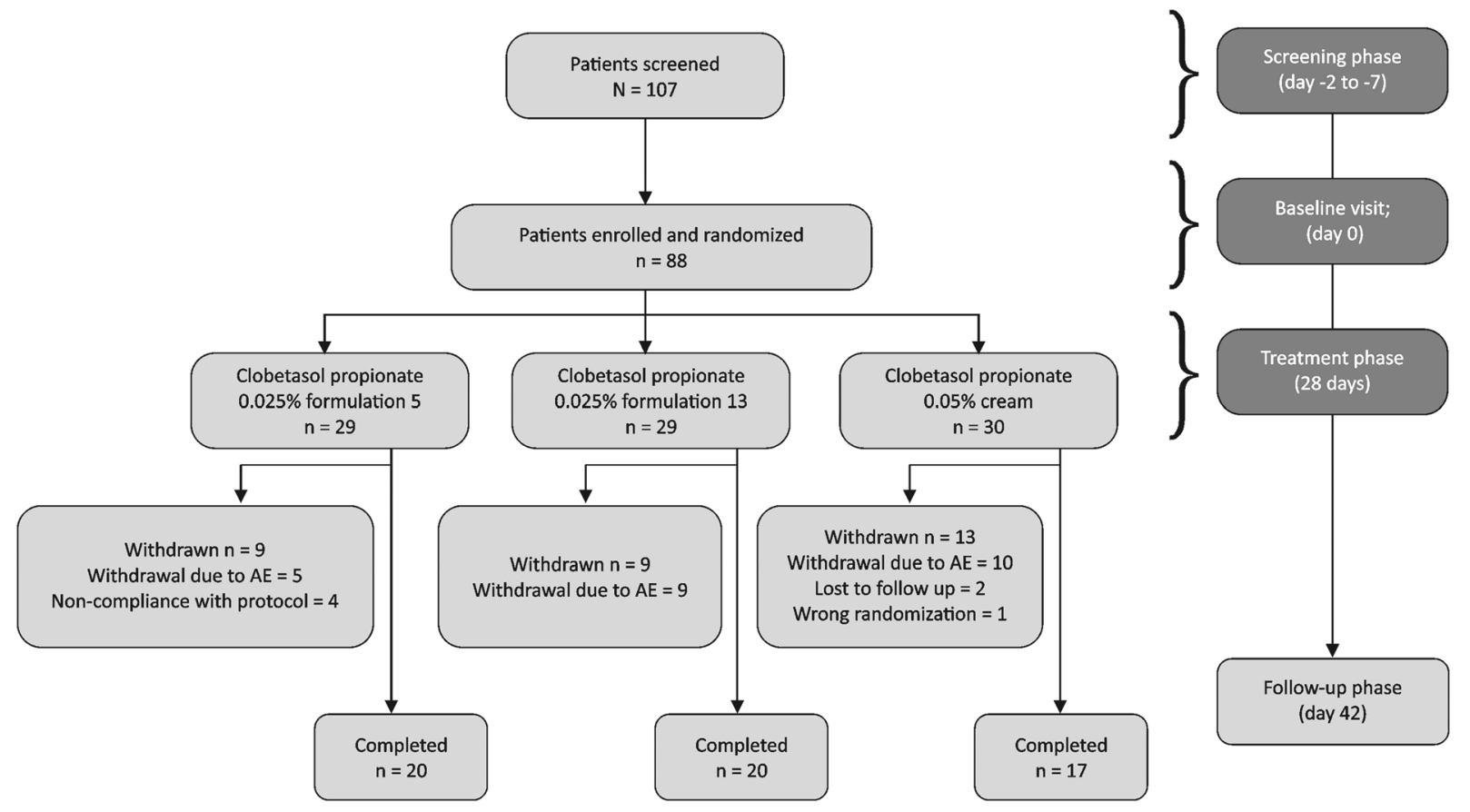

Fig. 1 Study design and patient characteristics. $A E$, adverse events 
Table 1 Patient demographic (safety population)

\begin{tabular}{|c|c|c|c|c|}
\hline \multirow[t]{2}{*}{ Characteristics } & \multicolumn{2}{|c|}{ Clobetasol propionate $0.025 \%$ cream } & \multirow{2}{*}{$\begin{array}{l}\text { Clobetasol propionate } \\
\mathbf{0 . 0 5 \%} \text { cream } \\
(N=30)\end{array}$} & \multirow{2}{*}{$\begin{array}{l}\text { All } \\
(N=88)\end{array}$} \\
\hline & $\begin{array}{l}\text { Formulation } 5 \\
(N=29)\end{array}$ & $\begin{array}{l}\text { Formulation } 13 \\
(N=29)\end{array}$ & & \\
\hline \multicolumn{5}{|l|}{ Age (years) } \\
\hline Mean (SD) & $44.1(16.88)$ & $47.0(13.49)$ & $39.1(10.51)$ & $43.3(14.06)$ \\
\hline \multicolumn{5}{|l|}{ Gender } \\
\hline Women, $n(\%)$ & $10(34.5)$ & $11(37.9)$ & $11(36.7)$ & $32(36.4)$ \\
\hline Men, $n(\%)$ & $19(65.5)$ & $18(62.1)$ & $19(63.3)$ & $56(63.6)$ \\
\hline \multicolumn{5}{|l|}{ Race } \\
\hline Asian, $n(\%)$ & $29(100.0)$ & $29(100.0)$ & $30(100.0)$ & $88(100.0)$ \\
\hline \multicolumn{5}{|l|}{ Height $(\mathrm{cm})$} \\
\hline Mean (SD) & $163.7(8.37)$ & $164.3(8.02)$ & $163.0(6.47)$ & $163.6(7.58)$ \\
\hline \multicolumn{5}{|l|}{ Weight $(\mathrm{Kg})$} \\
\hline Mean (SD) & $59.9(9.69)$ & $64.8(11.32)$ & $61.4(11.49)$ & $62.0(10.93)$ \\
\hline
\end{tabular}

$S D$ standard deviation.

products by the study label. The investigator was blinded to the treatment code until database lock.

\section{Statistical Analysis}

A total of 90 patients were planned to be randomized (1:1:1) to clobetasol propionate $0.025 \%$ formulation 5 , formulation 13 , or clobetasol propionate $0.05 \%$ cream. Efficacy assessments were analyzed in the per-protocol (PP; defined as all randomized patients who completed both baseline and end of treatment visit and who had no major protocol violations) and modified intention-to-treat (mITT; all randomized patients who received study medication and had at least one post randomization measurement) populations. Safety assessments were conducted in the safety population (all patients randomized at baseline visit who received at least one dose of study medication). The SAS ${ }^{\circledR}$ program (SAS ${ }^{\circledR}$ Institute Inc., USA, and Version 9.2) was used for statistical evaluation. The proportion of patients with abnormal ACTH stimulation and with clear or almost clear PGA at each time point was compared between groups using a chi-square test (Fisher's exact test). The Marascuillo procedure was used for pair-wise comparisons (clobetasol propionate $0.025 \%$ formulation 5 vs clobetasol propionate $0.05 \%$ cream and clobetasol propionate $0.025 \%$ formulation 13 vs clobetasol propionate $0.05 \%$ cream).

\section{RESULTS}

\section{Patient Disposition and Baseline Characteristics}

This study was conducted at six centers in India between April 2011 and October 2011. A total of 107 patients were screened, of which 88 were enrolled and randomized to clobetasol propionate $0.025 \%$ formulation $5(n=29)$, clobetasol propionate $0.025 \%$ formulation $13(n=29)$ and clobetasol propionate $0.05 \%$ cream $(n=30)$, of which 57 (64.8\%) patients completed the study (Fig. 1). The major reason for screen failure was patients not meeting the study selection criteria 


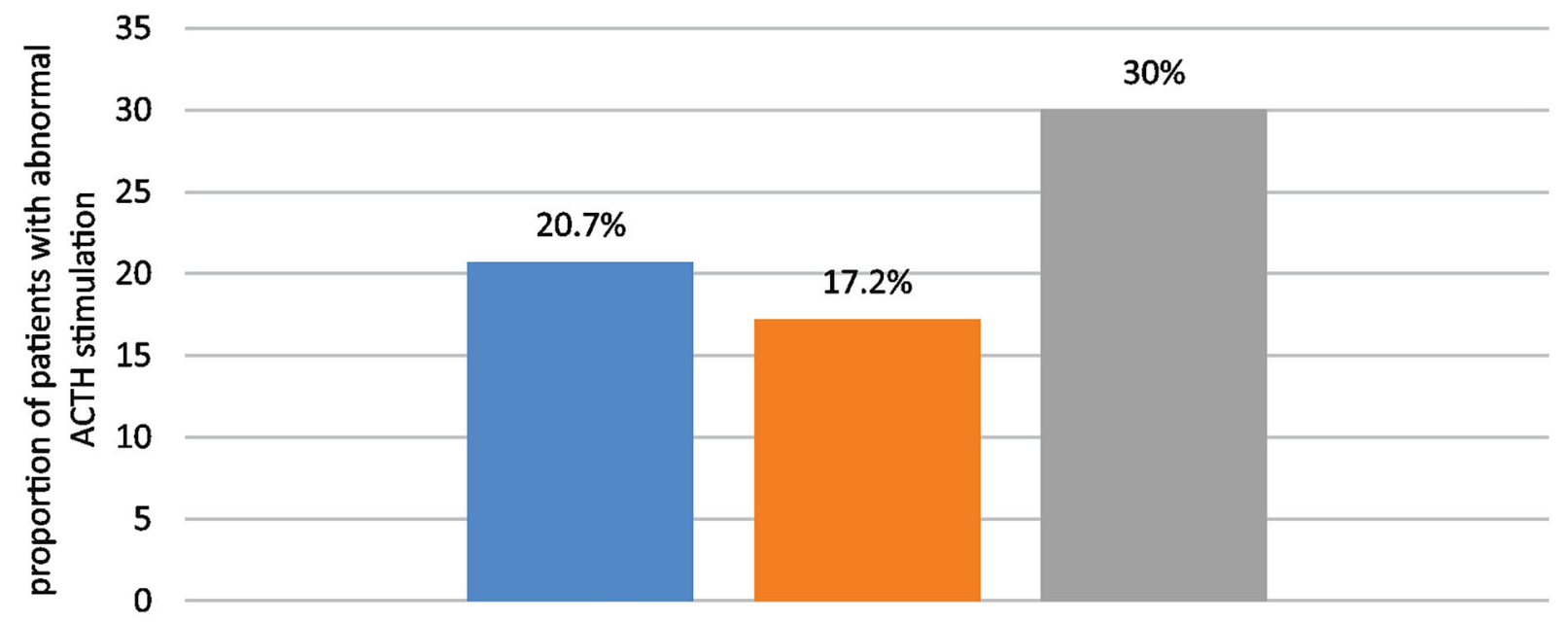

$$
\begin{aligned}
& \text { Clobetasol propionate } 0.025 \% \text { formulation } 5(n=29) \\
& \text { Clobetasol propionate } 0.025 \% \text { formulation } 13(n=29) \\
& \text { Clobetasol propionate } 0.05 \% \text { cream }(n=30)
\end{aligned}
$$

\begin{tabular}{|c|c|c|c|c|c|}
\hline Visit & ACTH result & $\begin{array}{l}\text { Clobetasol } \\
\text { propionate } \\
0.025 \% \\
\text { formulation } 5 \\
n=29\end{array}$ & $\begin{array}{l}\text { Clobetasol } \\
\text { propionate } \\
0.025 \% \\
\text { formulation } 13 \\
n=29\end{array}$ & $\begin{array}{c}\text { Clobetasol } \\
\text { propionate } \\
0.05 \% \\
\text { cream } \\
\mathbf{n}=\mathbf{3 0}\end{array}$ & P-value \\
\hline \multirow[b]{2}{*}{ Screening } & Abnormal & $1(3.4)$ & 0 & 0 & \\
\hline & Normal & $28(96.6)$ & $29(100)$ & $30(100)$ & \multirow{3}{*}{0.320} \\
\hline \multirow{2}{*}{$\begin{array}{l}\text { Visit } 6 \\
\text { (day 28) } \\
\end{array}$} & Abnormal & $6(20.7)$ & $5(17.2)$ & $9(30.0)$ & \\
\hline & Normal & $20(69.0)$ & $24(82.8)$ & $17(56.7)$ & \\
\hline \multicolumn{2}{|c|}{$\begin{array}{l}\text { Time to abnormal cortisol, } \\
\text { mean (SE) }\end{array}$} & $23.2(1.3)$ & $17.5(1.3)$ & $12.3(0.7)$ & 0.592 \\
\hline
\end{tabular}

Fig. 2 Proportion of patients with abnormal ACTH stimulation at day 28 (per protocol population). All values are presented in $n$ (\%). $A C T H$, adrenocorticotropic hormone; $S E$, standard error

or patients not having appropriate medicationspecific washout at the baseline visit. Of the 31 patients $(35.2 \%)$ who discontinued the study, $77.4 \%$ withdrew due to AEs [clobetasol propionate $0.025 \%$ formulation $5(n=5)$, clobetasol propionate $0.025 \%$ formulation $13(n=9)$ and clobetasol propionate $0.05 \%$ cream $(n=10)]$ (Fig. 1). The baseline demographics and clinical characteristics of all enrolled patients were comparable across treatment groups. The majority of patients were men $(63.6 \%)$, and all patients were Asians with a mean age of 43.3 years (Table 1 ).

\section{Safety}

At day 28, the proportion of patients with an abnormal ACTH stimulation test (cortisol levels $\leq 18 \mu \mathrm{g} / \mathrm{dl}$ ) was numerically lower in the clobetasol propionate $0.025 \%$ formulation 5 $(20.7 \%)$, and formulation $13(17.2 \%)$ groups, compared with the clobetasol propionate $0.05 \%$ 
Table 2 Incidence of treatment-emergent adverse events (safety population)

\begin{tabular}{|c|c|c|c|c|c|c|c|c|c|c|c|c|c|c|c|}
\hline \multirow[t]{3}{*}{ TEAEs, $n(\%)$} & \multicolumn{12}{|c|}{ Clobetasol propionate $0.025 \%$ cream } & \multirow{2}{*}{\multicolumn{3}{|c|}{$\begin{array}{l}\text { Clobetasol propionate } \\
0.05 \% \text { cream } \\
(N=30)\end{array}$}} \\
\hline & \multicolumn{6}{|c|}{ Formulation $5(N=29)$} & \multicolumn{6}{|c|}{ Formulation $13(N=29)$} & & & \\
\hline & \multicolumn{2}{|c|}{ Total } & \multicolumn{2}{|c|}{$\begin{array}{l}\text { Treatment } \\
\text { related }\end{array}$} & \multicolumn{2}{|c|}{$\begin{array}{l}\text { Probably } \\
\text { related }\end{array}$} & \multicolumn{2}{|c|}{ Total } & \multicolumn{2}{|c|}{$\begin{array}{l}\text { Treatment } \\
\text { related }\end{array}$} & \multicolumn{2}{|c|}{$\begin{array}{l}\text { Probably } \\
\text { related }\end{array}$} & Total & $\begin{array}{l}\text { Treatment } \\
\text { related }\end{array}$ & $\begin{array}{l}\text { Probably } \\
\text { related }\end{array}$ \\
\hline $\begin{array}{l}\text { Patients with at least } 1 \\
\text { TEAE }\end{array}$ & \multicolumn{2}{|c|}{$\begin{array}{l}13 \\
(44.8)\end{array}$} & 0 & & \multicolumn{2}{|c|}{$12(41.4)$} & \multicolumn{3}{|c|}{${ }_{(50.0)}^{15} 0$} & & \multicolumn{2}{|c|}{$10(34.5)$} & $\begin{array}{l}15 \\
\quad(50.0)\end{array}$ & 0 & $11(36.7)$ \\
\hline $\begin{array}{l}\text { TEAEs in }>3 \% \text { of } \\
\text { patients }\end{array}$ & \multicolumn{2}{|l|}{0} & \multicolumn{2}{|l|}{0} & \multicolumn{2}{|l|}{0} & \multicolumn{2}{|l|}{0} & \multicolumn{2}{|l|}{0} & \multicolumn{2}{|l|}{0} & $2(6.7)$ & 0 & 0 \\
\hline Pain & \multicolumn{2}{|l|}{0} & \multicolumn{2}{|l|}{0} & \multicolumn{2}{|l|}{0} & \multicolumn{2}{|l|}{0} & \multicolumn{2}{|l|}{0} & \multicolumn{2}{|l|}{0} & $1(3.3)$ & 0 & 0 \\
\hline Pyrexia & 0 & & 0 & & 0 & & 0 & & 0 & & 0 & & $1(3.3)$ & 0 & 0 \\
\hline Gastroenteritis & 0 & & 0 & & 0 & & 0 & & 0 & & 0 & & $1(3.3)$ & 0 & 0 \\
\hline Nasopharyngitis & 0 & & 0 & & 0 & & 0 & & 0 & & 0 & & $2(6.7)$ & 0 & 0 \\
\hline Tinea cruris & 0 & & 0 & & 0 & & 0 & & 0 & & 0 & & $1(3.3)$ & 0 & 0 \\
\hline Blood cortisol decrease & 13 & t.8) & 0 & & 12( & 1.4) & 11 & $37.9)$ & 0 & & & $(34.5)$ & $\begin{array}{l}11 \\
(36.7)\end{array}$ & 0 & $11(36.7)$ \\
\hline Cough & 0 & & 0 & & 0 & & 0 & & 0 & & 0 & & $2(6.7)$ & 0 & 0 \\
\hline Pruritus & $1(3$ & & 0 & & 0 & & 0 & & 0 & & 0 & & $1(3.3)$ & 0 & 0 \\
\hline Telangiectasia & 0 & & 0 & & 0 & & 0 & & 0 & & 0 & & $1(3.3)$ & 0 & $1(3.3)$ \\
\hline & & Mi & & Mod & erate & Sev & & Mil & & Modera & & Severe & Mild & Moderate & Severe \\
\hline Proportion of patients wi & mild & 11 & derate, & nd $s$ & & & & & & & & & & & \\
\hline Patients with at least 1 & EAE & & $(37.9)$ & $2(6$. & & 1( & & $7(2$ & & $3(10.3)$ & & $1(3.4)$ & $9(30.0)$ & $6(20.0)$ & $3(10.0)$ \\
\hline Pain & & 0 & & 0 & & 0 & & 0 & & 0 & & 0 & 0 & 0 & $1(3.3)$ \\
\hline Pyrexia & & 0 & & 0 & & 0 & & 0 & & 0 & & 0 & $1(3.3)$ & 0 & 0 \\
\hline Gastroenteritis & & 0 & & 0 & & 0 & & 0 & & 0 & & 0 & $1(3.3)$ & 0 & 0 \\
\hline Nasopharyngitis & & 0 & & 0 & & 0 & & 0 & & 0 & & 0 & 0 & $2(6.7)$ & 0 \\
\hline Tinea cruris & & 0 & & 0 & & 0 & & 0 & & 0 & & 0 & $1(3.3)$ & 0 & 0 \\
\hline Blood cortisol decrease & & 10 & $(34.5)$ & $2(6$. & & 1( & $.4)$ & $7(2$ & & $3(10.3)$ & & $1(3.4)$ & $6(20.0)$ & $4(13.3)$ & $1(3.3)$ \\
\hline Hyperglycemia & & 1( & 3.4) & 0 & & 0 & & 0 & & 0 & & 0 & 0 & 0 & 0 \\
\hline Cough & & 0 & & 0 & & 0 & & 0 & & 0 & & 0 & $2(6.7)$ & 0 & 0 \\
\hline Pruritus & & & $3.4)$ & 0 & & 0 & & 0 & & 0 & & 0 & 0 & 0 & $1(3.3)$ \\
\hline Telangiectasia & & 0 & & 0 & & 0 & & 0 & & 0 & & 0 & $1(3.3)$ & 0 & 0 \\
\hline
\end{tabular}

TEAEs treatment-emergent adverse events. 
Table 3 Change from baseline in signs of atrophogenicity by visit (safety population)

\begin{tabular}{|c|c|c|c|c|c|c|c|c|c|c|c|c|c|c|}
\hline \multirow[t]{3}{*}{ Treatment } & \multirow{3}{*}{$\begin{array}{l}\text { Baseline } \\
\text { atrophy score }\end{array}$} & \multicolumn{13}{|c|}{ Change from baseline in signs of atrophogenicity by visit: Atrophy score } \\
\hline & & \multicolumn{5}{|c|}{ Visit 3 (day 7), $n$ (\%) } & \multicolumn{3}{|c|}{ Visit 4 (day 14), $n$ (\%) } & \multicolumn{5}{|c|}{ Visit 5 (day 21), $n$ (\%) } \\
\hline & & \multicolumn{2}{|l|}{$\mathbf{0}$} & \multicolumn{2}{|l|}{1} & 2 & $\mathbf{0}$ & 1 & 2 & $\mathbf{0}$ & \multicolumn{2}{|l|}{1} & \multicolumn{2}{|l|}{2} \\
\hline \multirow{3}{*}{$\begin{array}{l}\text { Clobetasol propionate } \\
0.025 \% \text { formulation } 5\end{array}$} & $0(n=26)$ & \multicolumn{2}{|l|}{25} & \multicolumn{2}{|l|}{0} & 0 & 20 & $1(3.8)$ & 0 & 17 & \multicolumn{2}{|c|}{$2(7.7)$} & \multicolumn{2}{|l|}{0} \\
\hline & \multicolumn{6}{|c|}{$(96.2)$} & \multicolumn{2}{|c|}{$(76.9)$} & \multicolumn{4}{|c|}{$(65.4)$} & & \\
\hline & $1(n=3)$ & 0 & & $3(1$ & $00)$ & 0 & 0 & $3(100.0)$ & 0 & $1(33.3)$ & & 66.7) & 0 & \\
\hline \multirow{4}{*}{$\begin{array}{l}\text { Clobetasol propionate } \\
0.025 \% \text { formulation } 13\end{array}$} & $0(n=23)$ & \multirow{2}{*}{\multicolumn{2}{|c|}{$\begin{array}{l}22 \\
(95.7)\end{array}$}} & \multirow{2}{*}{\multicolumn{2}{|c|}{0}} & 0 & 15 & \multirow[t]{2}{*}{$2(8.7)$} & 0 & 12 & \multirow{2}{*}{\multicolumn{2}{|c|}{$3(13.0)$}} & \multirow{2}{*}{\multicolumn{2}{|c|}{0}} \\
\hline & & & & & & & $(65.2)$ & & & $(52.2)$ & & & & \\
\hline & $1(n=5)$ & \multicolumn{2}{|c|}{$1(20.0)$} & \multicolumn{2}{|c|}{$4(80.0)$} & 0 & $4(80.0)$ & $1(20.0)$ & 0 & $4(80.0)$ & \multicolumn{2}{|c|}{$1(20.0)$} & \multicolumn{2}{|l|}{0} \\
\hline & $2(n=1)$ & \multicolumn{2}{|l|}{0} & 0 & & $1(100)$ & $1(100)$ & 0 & 0 & $1(100)$ & 0 & & 0 & \\
\hline Clobetasol propionate & $0(n=26)$ & 22 & & 0 & & 0 & 16 & $1(3.8)$ & 0 & 11 & & $7.7)$ & & $(3.8)$ \\
\hline $0.05 \%$ cream & & & 34.6) & & & & $(61.5)$ & & & $(42.3)$ & & & & \\
\hline & $1(n=4)$ & 1( & 5.0) & $3(7$ & $5.0)$ & 0 & $2(50)$ & $2(50.0)$ & 0 & $4(100)$ & 0 & & 0 & \\
\hline Treatment & Baseline & & Chan & ge fr & m b & aseline in & signs of atr & rophogenici & ity by & y visit: $\mathrm{Te}$ & lan & giectas & ia sc & ore \\
\hline & telangiectasia sco & & $\overline{\text { Visit }}$ & $3(d$ & y 7), & $n(\%)$ & Visit 4 (c & day 14), $n($ & (\%) & Visit 5 & (da & 21), & $n(\%$ & \\
\hline & & & 0 & & 1 & 2 & $\mathbf{0}$ & 1 & 2 & $\mathbf{0}$ & & 1 & & 2 \\
\hline $\begin{array}{l}\text { Clobetasol propionate } \\
0.025 \% \text { formulation } 5\end{array}$ & $0(n=29)$ & & $28(9$ & $6.6)$ & 0 & 0 & $24(82.8)$ & 0 & 0 & $20(69.0$ & & $2(6.9$ & & 0 \\
\hline Clobetasol propionate & $0(n=28)$ & & $27(9$ & $6.4)$ & 0 & 0 & $21(75.0)$ & $1(3.6)$ & 0 & $17(60.7$ & & $3(10$ & & 0 \\
\hline & $2(n=1)$ & & 0 & & 0 & $1(100)$ & $1(100)$ & 0 & 0 & $1(100)$ & & 0 & & 0 \\
\hline TMV cream & $0(n=30)$ & & $26(8$ & 6.7) & 0 & 0 & $21(70.0)$ & 0 & 0 & $16(53.3$ & & $2(6.7$ & & 0 \\
\hline
\end{tabular}

0 , none; 1 , slight; 2 , moderate; 3 , pronounced; 4 , very pronounced.

cream (30.0\%) group, though the differences were not statistically significant $(p=0.320)$ (Fig. 2). The mean (SE) time to abnormal cortisol value was longer in the clobetasol propionate $0.025 \%$ formulation 5 group [23.2 (1.3) days] and formulation 13 group [17.5 (1.3) days] when compared with the clobetasol propionate $0.05 \%$ cream group [12.3 (0.7) days], though the differences were not statistically significant $(p=0.592)$. Overall, $44.8 \%(13 / 29)$ of patients in the clobetasol propionate $0.025 \%$ formulation 5 group, $50.0 \%(15 / 29)$ in the formulation 13 group, and $50 \%(15 / 30)$ of patients in clobetasol propionate $0.05 \%$ cream group experienced at least 1 treatment-emergent adverse event (TEAE) (Table 2). None of the AEs were considered by the investigator as definitely related to the treatment. Decreased blood cortisol was the common AE reported in both the clobetasol propionate $0.025 \%$ formulation 5 (44.8\%) and formulation 13 (37.9\%) groups, and also in the clobetasol propionate $0.05 \%$ cream group (36.7\%). Most of the AEs reported were mild in severity $(37.9 \%$ in the clobetasol propionate $0.025 \%$ formulation 5 group, $24.1 \%$ in the clobetasol propionate $0.025 \%$ formulation 13 group, and $30.0 \%$ in the clobetasol propionate $0.05 \%$ cream group). Severe AEs were reported in $3.4 \%$ of patients in both the clobetasol propionate $0.025 \%$ formulation 5 and 13 groups, and $10 \%$ of patients in the clobetasol propionate $0.05 \%$ cream group. Overall, 
Table 4 Proportion of patients with burning/stinging and pruritus scores by visit (safety population)

\begin{tabular}{|c|c|c|c|}
\hline \multirow[t]{2}{*}{ Burning/stinging, $n$ (\%) } & \multicolumn{2}{|c|}{ Clobetasol propionate $0.025 \%$ cream } & \multirow{2}{*}{$\begin{array}{l}\text { Clobetasol propionate } \\
0.05 \% \text { cream } \\
(N=30)\end{array}$} \\
\hline & $\begin{array}{l}\text { Formulation } 5 \\
(N=29)\end{array}$ & $\begin{array}{l}\text { Formulation } 13 \\
(N=29)\end{array}$ & \\
\hline \multicolumn{4}{|l|}{ Visit 3 (day 7) } \\
\hline 0 & $10(34.5)$ & $12(41.4)$ & $9(30.0)$ \\
\hline 1 & $11(37.9)$ & $13(44.8)$ & $14(46.7)$ \\
\hline 2 & $5(17.2)$ & $3(10.3)$ & $2(6.7)$ \\
\hline 3 & $2(6.9)$ & 0 & $1(3.3)$ \\
\hline \multicolumn{4}{|l|}{ Visit 4 (day 14) } \\
\hline 0 & $9(31.0)$ & $13(44.8)$ & $11(36.7)$ \\
\hline 1 & $13(44.8)$ & $9(31.0)$ & $10(33.3)$ \\
\hline 2 & $2(6.9)$ & $1(3.4)$ & 0 \\
\hline \multicolumn{4}{|l|}{ Visit 5 (day 21) } \\
\hline 0 & $9(31.0)$ & $16(55.2)$ & $12(40.0)$ \\
\hline 1 & $12(41.4)$ & $5(17.2)$ & $6(20.0)$ \\
\hline 2 & $1(3.4)$ & 0 & 0 \\
\hline \multicolumn{4}{|l|}{ Visit 6 (day 28) } \\
\hline 0 & $15(51.7)$ & $20(69.0)$ & $21(70.0)$ \\
\hline 1 & $10(34.5)$ & $7(24.1)$ & $6(20.0)$ \\
\hline 2 & $1(3.4)$ & $2(6.9)$ & 0 \\
\hline
\end{tabular}

Pruritus, $n(\%)$

Clobetasol propionate $\mathbf{0 . 0 2 5 \%}$ cream

Formulation 5

$(N=29)$

Formulation 13

$(N=29)$

8 (27.6)

$12(41.4)$

8 (27.6)

0
7 (24.1)
$15(51.7)$
1 (3.4)

0

$\begin{array}{ll}6(20.7) & 7(24.1) \\ 16(55.2) & 15(51.7) \\ 2(6.9) & 1(3.4) \\ 0 & 0\end{array}$

7 (23.3)

$12(40.0)$

1 (3.3)

$1(3.3)$ $(N=30)$ $\mathbf{0 . 0 5 \%}$ cream

Visit 5 (day 21) 
Table 4 continued

\begin{tabular}{|c|c|c|c|}
\hline \multirow[t]{2}{*}{ Pruritus, $n(\%)$} & \multicolumn{2}{|c|}{ Clobetasol propionate $0.025 \%$ cream } & \multirow{2}{*}{$\begin{array}{l}\text { Clobetasol propionate } \\
0.05 \% \operatorname{cream}(N=30)\end{array}$} \\
\hline & $\begin{array}{l}\text { Formulation } \\
5(N=29)\end{array}$ & $\begin{array}{l}\text { Formulation } \\
13(N=29)\end{array}$ & \\
\hline 0 & $8(27.6)$ & $12(41.1)$ & $10(33.3)$ \\
\hline 1 & $11(37.9)$ & $9(31.0)$ & $6(20.0)$ \\
\hline 2 & $3(10.3)$ & 0 & $1(3.3)$ \\
\hline 3 & 0 & 0 & $1(3.3)$ \\
\hline \multicolumn{4}{|l|}{ Visit 6 (day 28) } \\
\hline 0 & $11(37.9)$ & $15(51.7)$ & $14(46.7)$ \\
\hline 1 & $13(44.8)$ & $11(37.9)$ & $11(36.7)$ \\
\hline 2 & $2(6.9)$ & $3(10.3)$ & $2(6.7)$ \\
\hline
\end{tabular}

0 , none; 3 , severe.

5 patients in the clobetasol propionate $0.025 \%$ formulation 5 group, 9 patients in the clobetasol propionate $0.025 \%$ formulation 13 group, and 10 patients in the clobetasol propionate $0.05 \%$ cream group withdrew from the study due to decreased blood cortisol. No serious AEs or deaths were reported during the study.

No shift in atrophy scores from baseline to day 7 was observed in the clobetasol propionate $0.025 \%$ formulation 5 group; however, a shift in atrophy score from 1 to 0 from baseline to visit 3 for 1 patient each in the clobetasol propionate $0.025 \%$ formulation 13 group and the clobetasol propionate $0.05 \%$ cream group (Table 3 ) was noted. No change in telangiectasia score from baseline to visit 3 in any of the treatment groups was observed. A shift in telangiectasia score from 2 to $0(n=1)$ and 0 to $1(n=1)$ from baseline to visit 4 in the clobetasol propionate $0.025 \%$ formulation 13 group was observed. Additionally, a shift from baseline to visit 5 in telangiectasia score of 0 to $1(n=2)$ in the clobetasol propionate $0.025 \%$ formulation 5 group, a shift of 0 to $1(n=3)$ and 2 to $0(n=1)$ in the clobetasol propionate $0.025 \%$ formulation 13 group, and a shift from 0 to $1(n=2)$ in the clobetasol propionate $0.05 \%$ cream group was observed (Table 3).
A gradual decrease in the scores of burning/ stinging in all three groups was observed by day 28 (clobetasol propionate $0.025 \%$ formulation 5 vs clobetasol propionate $0.025 \%$ formulation 13 vs clobetasol propionate $0.05 \%$ cream groups, score 0: $51.7 \%, 69.0 \%$, and $70.0 \%$; score 1 : $34.5 \%, 24.1 \%$, and $20 \%$; score $2: 3.4 \%, 6.9 \%$, and none). The treatment groups were comparable with respect to burning/stinging scores at all visits. Similarly, a reduction in the pruritus score was noted at visit 6 (clobetasol propionate $0.025 \%$ formulation 5 vs clobetasol propionate $0.025 \%$ formulation 13 vs clobetasol propionate $0.05 \%$ cream groups, score $0: 37.9 \%, 51.7 \%$, and $46.7 \%$; score 1: $44.8 \%, 37.9 \%$, and $36.7 \%$; score 2: $6.9 \%, 10.3 \%$, and $6.7 \%)$. The three groups were comparable with respect to pruritus scores at all visits (Table 4).

\section{Efficacy}

In the PP population, at the baseline visit the proportion of patients with a PGA score of 3 were $61.1 \%, 63.2 \%$, and $53.8 \%$ and those with a PGA score of 4 were $38.9 \%, 36.8 \%$, and $46.2 \%$ in clobetasol propionate $0.025 \%$ formulation 5 , clobetasol propionate $0.025 \%$ formulation 13 , and clobetasol propionate $0.05 \%$ cream groups, respectively. The mean PGA score gradually 
Table 5 Proportion of patients with PGA score by visit (per protocol population)

\begin{tabular}{|c|c|c|c|}
\hline \multirow[t]{2}{*}{ PGA score by visit } & \multicolumn{2}{|c|}{ Clobetasol propionate $0.025 \%$ cream } & \multirow{2}{*}{$\begin{array}{l}\text { Clobetasol propionate } 0.05 \% \text { crean } \\
(N=13)\end{array}$} \\
\hline & $\begin{array}{l}\text { Formulation } 5 \\
(N=18)\end{array}$ & $\begin{array}{l}\text { Formulation } 13 \\
(N=19)\end{array}$ & \\
\hline \multicolumn{4}{|l|}{ Baseline visit } \\
\hline 0 & 0 & 0 & 0 \\
\hline 1 & 0 & 0 & 0 \\
\hline 2 & 0 & 0 & 0 \\
\hline 3 & $11(61.1)$ & $12(63.2)$ & $7(53.8)$ \\
\hline 4 & $7(38.9)$ & $7(36.8)$ & $6(46.2)$ \\
\hline Mean (SD) & $3.4(0.50)$ & $3.4(0.50)$ & $3.5(0.52)$ \\
\hline Success & 0 & 0 & 0 \\
\hline \multicolumn{4}{|l|}{ Visit 3 (day 7) } \\
\hline 0 & 0 & 0 & 0 \\
\hline 1 & 0 & 0 & 0 \\
\hline 2 & 0 & $3(15.8)$ & $1(7.7)$ \\
\hline 3 & $14(77.8)$ & $13(68.4)$ & $9(69.2)$ \\
\hline 4 & $4(22.2)$ & $3(15.8)$ & $3(23.1)$ \\
\hline Mean (SD) & $3.2(0.43)$ & $3.0(0.58)$ & $3.2(0.55)$ \\
\hline Success & 0 & 0 & 0 \\
\hline \multicolumn{4}{|l|}{ Visit 4 (day 14) } \\
\hline 0 & 0 & 0 & 0 \\
\hline 1 & 0 & $1(5.3)$ & $1(7.7)$ \\
\hline 2 & $8(44.4)$ & $9(47.4)$ & $5(38.5)$ \\
\hline 3 & $10(55.6)$ & $7(36.8)$ & $7(53.8)$ \\
\hline 4 & 0 & $2(10.5)$ & 0 \\
\hline Mean (SD) & $2.6(0.51)$ & $2.5(0.77)$ & $2.5(0.66)$ \\
\hline Success $^{\mathrm{a}}$ & 0 & $1(5.3)$ & $1(7.7)$ \\
\hline \multicolumn{4}{|l|}{ Visit 5 (day 21) } \\
\hline 0 & 0 & $1(5.3)$ & $1(7.7)$ \\
\hline 1 & $2(11.1)$ & $1(5.3)$ & 0 \\
\hline 2 & $13(72.2)$ & $13(68.4)$ & $10(76.9)$ \\
\hline 3 & $3(16.7)$ & $4(21.1)$ & $2(15.4)$ \\
\hline 4 & 0 & 0 & 0 \\
\hline Mean (SD) & $2.1(0.54)$ & $2.1(0.71)$ & $2.0(0.71)$ \\
\hline
\end{tabular}


Table 5 continued

\begin{tabular}{|c|c|c|c|}
\hline \multirow[t]{2}{*}{ PGA score by visit } & \multicolumn{2}{|c|}{ Clobetasol propionate $0.025 \%$ cream } & \multirow{2}{*}{$\begin{array}{l}\text { Clobetasol propionate } 0.05 \% \\
\operatorname{cream}(N=13)\end{array}$} \\
\hline & $\begin{array}{l}\text { Formulation } \\
5(N=18)\end{array}$ & $\begin{array}{l}\text { Formulation } \\
13(N=19)\end{array}$ & \\
\hline Success ${ }^{\mathrm{b}}$ & $2(11.1)$ & $2(10.5)$ & $1(7.7)$ \\
\hline \multicolumn{4}{|l|}{ Visit 6 (day 28) } \\
\hline 0 & 0 & $1(5.3)$ & $1(7.7)$ \\
\hline 1 & $7(38.9)$ & $6(31.6)$ & $3(23.1)$ \\
\hline 2 & $11(61.1)$ & $10(52.6)$ & $8(61.5)$ \\
\hline 3 & 0 & $2(10.5)$ & $1(7.7)$ \\
\hline 4 & 0 & 0 & 0 \\
\hline Mean (SD) & $1.6(0.50)$ & $1.7(0.75)$ & $1.7(0.75)$ \\
\hline Success $^{\mathrm{c}}$ & $7(38.9)$ & $7(36.8)$ & $4(30.8)$ \\
\hline
\end{tabular}

All values are presented as $\mathrm{n}(\%)$ unless specified. $S D$, standard deviation.

0 , clear; 1 , almost clear; 2 , mild; 3 , moderate; 4 , moderate to severe.

${ }^{a} p$ value 0.5245

b $p$ value 0.9477

c $p$ value 0.8934

reduced from baseline visit to end of treatment (day 28) in all three treatment groups, with no significant differences between the groups [clobetasol propionate $0.025 \%$ formulation 5 (baseline to day 28: 3.4 to 1.6), clobetasol propionate $0.025 \%$ formulation 13 (baseline to day 28: 3.4 to 1.7 ), and clobetasol propionate $0.05 \%$ cream (baseline to day 28: 3.5 to 1.7)] (Table 5). The PGA success rates (clear or almost clear) at the end of the study were $38.9 \%, 36.8 \%$, and $30.8 \%$ in patients treated with clobetasol propionate $0.025 \%$ formulation 5 , clobetasol propionate $0.025 \%$ formulation 13 , and clobetasol propionate $0.05 \%$ cream, respectively. The PGA success rates were comparable across the three treatment groups with no significant differences $(p=0.893)$.

\section{DISCUSSION}

This comparative phase 2a study aimed to evaluate HPA axis suppression, safety, and efficacy of novel formulations of $0.025 \%$ clobetasol propionate test creams (clobetasol propionate $0.025 \%$ formulations 5 and 13) with the currently marketed $0.05 \%$ formulation of clobetasol propionate cream in patients with moderateto-severe psoriasis. The results of the present study demonstrated that both the new formulations, with half the concentration $(0.025 \%)$ of currently marketed clobetasol propionate $0.05 \%$ formulation, had a numerically lower risk of causing HPA axis suppression, while showing similar efficacy to the currently marketed full strength clobetasol propionate $0.05 \%$ product. There was a trend towards lower HPA axis suppression in the lower strength formulations compared with the higher strengths, but the study results lacked statistical significance. Also, the lower strength formulations caused a more delayed reduction in the endogenous cortisol levels: together, these aspects make for a better risk-benefit profile compared with the higher strength product. Formulation 5, which was selected for further studies, had a higher trend for mean (SE) time to abnormal cortisol value [23.2 (1.3) days] compared with clobetasol 


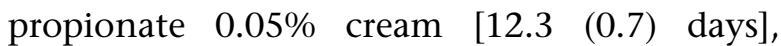
though the differences were not statistically significant $(p=0.592)$.

No patient had a PGA score of 4 after 3 weeks of treatment in any of the groups, demonstrating similar efficacy of the novel clobetasol propionate formulations $(0.025 \%)$ to that of clobetasol propionate $0.05 \%$ cream. The treatments with both test and reference formulations were effective, with PGA success rates achieved in $\sim 37.9 \%$ of patients in the clobetasol propionate $0.025 \%$ formulation groups, which was comparable with the reference clobetasol propionate $0.05 \%$ cream group $(30.8 \%)$ at the end of treatment. Though not significant ( $p$-value, 0.893 ), the proportion of patients with PGA success rates at day 28 were numerically higher in the clobetasol propionate $0.025 \%$ formulation groups compared with the clobetasol propionate $0.05 \%$ formulation group. The mean (SD) PGA score in patients with chronic psoriasis treated with once daily clobetasol propionate $0.05 \%$ cream was 2.46 (0.92) [18], while in our study the twice daily application of both clobetasol propionate $0.05 \%$ cream and clobetasol propionate $0.025 \%$ formulations for 4 weeks showed a numerically greater reduction in mean (SD) PGA scores at the end of the study favoring twice daily application of clobetasol propionate creams.

The topical use of clobetasol propionate at a dose as low as $2 \mathrm{~g} /$ day $(0.05 \%$ cream $)$ can cause HPA suppression within a few days. In a previous study, the frequency of HPA axis suppression was $\sim 40 \%$ in patients using $0.05 \%$ clobetasol propionate for $2-3$ weeks $[19,20]$. The novel formulation containing half the strength of clobetasol propionate, which is associated with decreased systemic absorption, could be a safer alternative to the currently marketed clobetasol propionate $0.05 \%$ cream. In a recent phase 2 study, which was mandated by USFDA for the approval of the novel formulation containing half the strength of clobetasol propionate cream (clobetasol propionate $0.025 \%$ ), the proportion of patients with abnormal ACTH stimulation (indicating HPA suppression) was lower in the clobetasol propionate $0.025 \%$ group (12.5\%) compared with the clobetasol propionate $0.05 \%$ cream group (36.4\%) after twice-daily treatment for 15 days [17]. In the present study, the proportion of patients with abnormal ACTH stimulation after 28 days of treatment was numerically lower in the clobetasol propionate $0.025 \%$ formulation groups compared with the clobetasol propionate $0.05 \%$ formulation group ( $p$-value, 0.320 ). Additionally, although not statistically significant, time to abnormal cortisol value was also longer in the clobetasol propionate $0.025 \%$ formulation groups compared with the $0.05 \%$ cream group, indicating that the reference product with double strength causes abnormality in cortisol homeostasis at a much earlier time point. These results should be interpreted cautiously as the present study had a small sample size; larger studies may be required to further validate these results. Further, our study is limited as the pharmacokinetics of the drug were not analyzed, which may limit the interpretation of plasma drug concentration correlation with HPA axis suppression. In a recent phase 2 comparative study in the US, it was reported that after 15 days of treatment, the plasma drug concentration of clobetasol propionate $0.025 \%$ was significantly less than that of clobetasol propionate $0.05 \%(56.3 \mathrm{pg} / \mathrm{ml}$ vs $152.5 \mathrm{pg} / \mathrm{ml}$; $p=0.014$ ) [17]. A correlation between lower systemic exposure and lower HPA axis suppression was also seen in this study, indicating a better risk-benefit profile for the clobetasol propionate $0.025 \%$ cream, compared with the currently marketed clobetasol propionate $0.05 \%$ cream.

The data demonstrated comparable results with respect to the safety profile of clobetasol propionate $0.05 \%$ cream. A decrease in blood cortisol concentration was the commonly reported AE responsible for patient withdrawal in all the treatment groups. Most of the AEs reported in the study were mild in severity and none of the AEs were considered to be definitely related to treatment by the investigator. No serious AEs or deaths were reported in this study. It is noteworthy that this study had a longer treatment duration of 4 weeks in comparison to other clinical studies of clobetasol propionate with recommended 2-week treatment duration in patients with psoriasis $[14,16,21,22]$. Despite the longer treatment 
duration, there were no new safety findings observed in any of the treatment groups.

An interesting observation was that the results pertaining to HPA axis suppression using clobetasol propionate $0.025 \%$ cream in this Indian study are not, in fact, statistically significant but showed similar efficacy to that of $0.05 \%$ strength. And because systemic exposure to steroids depends upon their potency, the novel half-strength formulation (0.025\%) offered a lower degree of systemic absorption of this potent topical corticosteroid without compromising on efficacy [17]. Additionally, no significant difference in baseline BSA between the groups was observed and the amount of formulation applied was calculated based on BSA to ensure that there was no inter-subject variability. The BSA and the amount of drug applied were not significantly different between the groups, which avoided bias. In line with Draelos et al.'s study, our study results confirmed that the half-strength (compared with marketed propionate cream) novel formulations $(0.025 \%$, formulation 5 and 13$)$ had similar efficacy to that of the $0.05 \%$ formulation. Hence, these reduced strength clobetasol propionate $0.025 \%$ formulations could serve as a better treatment option for patients with moderate-to-severe psoriasis, while avoiding exposure to higher concentrations of the drug that may result in long-term toxicities.

\section{Limitations}

The present study had a small sample size and further studies with a higher sample number may be required to validate these results. The pharmacokinetics of the drug were not analyzed in the present study, which may restrict the interpretation of plasma drug concentration correlation with HPA axis suppression.

\section{CONCLUSIONS}

The novel formulations of clobetasol propionate (formulations 5 and 13) had numerically, yet not significantly, lesser HPA axis suppression than the full-strength marketed product, while showing similar efficacy. Cortisol homeostasis seemed to be impacted earlier by the clobetasol propionate $0.05 \%$ cream compared with the clobetasol propionate $0.025 \%$ formulations as shown by 'Time to Abnormal Cortisol'. Overall, our study findings support the use of the novel clobetasol propionate $0.025 \%$ cream formulations (formulation 5 and formulation 13) as an effective and safer treatment option for patients with moderate-tosevere psoriasis. The novel formulations of clobetasol propionate $0.025 \%$ have similar efficacy and an improved safety profile compared with the currently marketed clobetasol propionate $0.05 \%$ formulation.

\section{ACKNOWLEDGEMENTS}

The authors thank the study patients without whom this study would not have been accomplished, and also thank the investigator site team for their support in this study.

Funding. Sponsorship for this study and the journal's Rapid Service Fee were funded by Dr. Reddy's Laboratories Ltd.

Medical Writing, Editorial, and Other Assistance. We thank Preethi Bheereddy M.S (Pharm) and Nigar Malik (B.Pharm), SIRO ClinPharm (funded by DRL) for writing, editorial and graphics support.

Authorship. All named authors meet the International Committee of Medical Journal Editors (ICMJE) criteria for authorship for this article, take responsibility for the integrity of the work as a whole, and have given their approval for this version to be published.

Author Contributions. S M Hussain Naqvi, R Mittal, S Mehta and A Mane drafted and critically reviewed the manuscript. S. Sidgiddi planned and executed the clinical study. M M Shenoy, D N Balraj. J Kothari, S Gupta and R Haq were the study investigators.

Disclosures. S. M. Hussain Naqvi, R. Mittal, S. Mehta, A. Mane are an employee of Dr. Reddy's Laboratories Inc. S. Sidgiddi is an employee 
of Dr. Reddy's Laboratories Inc. and owns stock in the company. MM. Shenoy, D. N. Balraj, J. Kothari, G. Sandesh, R. Haq received research fund from Dr. Reddy's Laboratories.

Compliance with Ethics Guidelines. The study protocol and informed consent form were reviewed and approved by an Independent Ethics Committee and Institutional Review Board at all six study sites (please see supplementary material for full details). The study was conducted in accordance with the ethical principles of the Declaration of Helsinki and per the International Council for Harmonization and Good Clinical Practice guidelines. Informed consent forms were reviewed and approved by all the appropriate Ethics Committees prior to enrolment of the patients into the study.

Data Availability. The datasets generated during and/or analyzed during the current study are available from the corresponding author on reasonable request.

Open Access. This article is licensed under a Creative Commons Attribution-NonCommercial 4.0 International License, which permits any non-commercial use, sharing, adaptation, distribution and reproduction in any medium or format, as long as you give appropriate credit to the original author(s) and the source, provide a link to the Creative Commons licence, and indicate if changes were made. The images or other third party material in this article are included in the article's Creative Commons licence, unless indicated otherwise in a credit line to the material. If material is not included in the article's Creative Commons licence and your intended use is not permitted by statutory regulation or exceeds the permitted use, you will need to obtain permission directly from the copyright holder. To view a copy of this licence, visit http:// creativecommons.org/licenses/by-nc/4.0/.

\section{REFERENCES}

1. Langley RG, Krueger GG, Griffiths CE. Psoriasis: epidemiology, clinical features, and quality of life. Ann Rheum Dis. 2005;64(Suppl 2):18-23.

2. Dogra S, Mahajan R. Psoriasis: epidemiology, clinical features, co-morbidities, and clinical scoring. Indian Dermatol Online J. 2016;7(6):471-80.

3. Dogra S, Yadav S. Psoriasis in India: prevalence and pattern. Indian J Dermatol Venereol Leprol. 2010;76(6):595-601.

4. Global report on Psoriasis. World Health Organization. 2016. Available from: https://apps.who.int/ iris/bitstream/handle/10665/204417/9789241565 189_eng.pdf.psoriasis; jsessionid=54912784D28C9 F36ECCD45471AC5775B?sequence $=1$. Accessed 04 Oct 2007.

5. Menter A, Gottlieb A, Feldman SR, Van Voorhees AS, Leonardi CL, Gordon KB, et al. Guidelines of care for the management of psoriasis and psoriatic arthritis: section 1. Overview of psoriasis and guidelines of care for the treatment of psoriasis with biologics. J Am Acad Dermatol. 2008;58(5):826-50.

6. Kim WB, Jerome D, Yeung J. Diagnosis and management of psoriasis. Can Fam Physician. 2017;63(4):278-85.

7. De Korte J, Mombers FM, Bos JD, Sprangers MA. Quality of life in patients with psoriasis: a systematic literature review. J Investig Dermatol Symp Proc. 2004;9(2):140-7.

8. Mrowietz U, Kragballe K, Reich K, Spuls P, Griffiths CE, Nast A, et al. Definition of treatment goals for moderate to severe psoriasis: a European consensus. Arch Dermatol Res. 2011;303(1):1-10.

9. Feldman SR, Duffin KC. Treatment of psoriasis in adults. UpToDate. 2017. https://www.uptodate. com/contents/treatment-of-psoriasis-in-adults. Accessed 03 Oct 2007.

10. Menter A, Korman NJ, Elmets CA, Feldman SR, Gelfand JM, Gordon KB, et al. Guidelines of care for the management of psoriasis and psoriatic arthritis: section 3. Guidelines of care for the management and treatment of psoriasis with topical therapies. J Am Acad Dermatol. 2009;60(4):643-59.

11. Samarasekera EJ, Sawyer L, Wonderling D, Tucker R, Smith $\mathrm{CH}$. Topical therapies for the treatment of plaque psoriasis: systematic review and network meta-analyses. Br J Dermatol. 2013;168(5):954-67.

12. Pariser D, Bukhalo $M$, Guenthner $S$, Kempers $S$, Shideler S, Gold LS, et al. Two multicenter, 
randomized, double-blind, parallel arm comparison studies of a novel enhanced lotion formulation of halobetasol propionate, $0.05 \%$ versus its vehicle in adult subjects with plaque psoriasis. J Drugs Dermatol. 2017;16(3):234-40.

13. Warino L, Balkrishnan R, Feldman SR. Clobetasol propionate for psoriasis: are ointments really more potent? J Drugs Dermatol. 2006;5(6):527-32.

14. Austad J, Bjerke JR, Gjertsen BT, Helland S, Livden JK, Morken T, et al. Clobetasol propionate followed by calcipotriol is superior to calcipotriol alone in topical treatment of psoriasis. J Eur Acad Dermatol Venereol. 1998;11(1):19-24.

15. Gilbertson EO, Spellman MC, Piacquadio DJ, Mulford MI. Super potent topical corticosteroid use associated with adrenal suppression: clinical considerations. J Am Acad Dermatol. 1998;38(2): 318-21.

16. Olsen EA, Cornell RC. Topical clobetasol-17-propionate: review of its clinical efficacy and safety. J Am Acad Dermatol. 1986;15(2):246-55.

17. Draelos ZD, Fowler JF, Cornelison R. A randomized, parallel group, open label, multicenter study to assess the potential for adrenal suppression and systemic drug absorption following multiple dosing with clobetasol propionate cream (Impoyz $\left.{ }^{\mathrm{TM}}\right), 0$. $025 \%$ versus clobetasol propionate (Temovate $^{\circledR}$ ). Skin. 2018. https://doi.org/10.25251/skin.2.6.16.

18. Goruntla N, Arakala GK, Nelluri GP, Mounika KN, Pujari S, Byalla MK. Comparison of efficacy, safety, and cost-effectiveness of topical salicylic acid $6 \%$ versus clobetasol propionate $0.05 \%$ in the treatment of limited chronic plaque psoriasis. J Health Res Rev. 2018;5(2):86-95.

19. Kerner M, Ishay A, Ziv M, Rozenman D, Luboshitzky R. Evaluation of the pituitary-adrenal axis function in patients on topical steroid therapy. J Am Acad Dermatol. 2011;65(1):215-6.

20. Muhammad AS, Majid H, Mansoor D. Frequency of serum cortisol suppression in patients using $0.05 \%$ clobetasol propionate topical steroid for more than 2 weeks. Pak Armed Forces Med J. 2016;66(2): 199-202.

21. Ellis CN, Scott EJ. Clobetasol propionate cream versus halcinonide cream in psoriasis. Int J Dermatol. 1986;25(5):332-3.

22. Bleeker J. Double-blind comparison between two new topical corticosteroids, halcinonide $0.1 \%$ and clobetasol propionate cream $0.05 \%$. Curr Med Res Opin. 1975;3(4):225-8. 